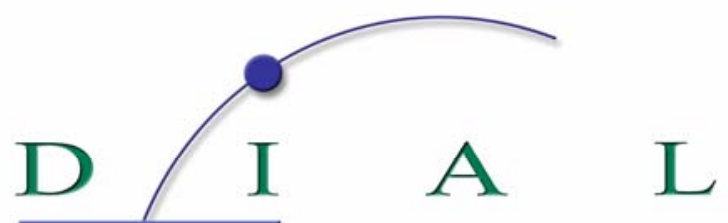

Développement Institutions \& Analyses de Long terme

DOCUMENT DE TRAVAIL

DT/2006-18

\title{
Impact des financements internationaux sur les inégalités des pays en développement
}

Lisa CHAUVET

Sandrine MESPLE-SOMPS 


\title{
IMPACT DES FINANCEMENTS INTERNATIONAUX SUR LES INEGALITES DES PAYS EN DEVELOPPEMENT
}

\author{
Lisa Chauvet \\ IRD, DIAL, Paris \\ chauvet@dial.prd.fr \\ Sandrine Mesplé-Somps \\ mesple@dial.prd.fr
}

Document de travail DIAL

Décembre 2006

\section{RESUME}

Nous proposons une analyse économétrique de l'impact sur la distribution des revenus de quatre sources de flux de financements internationaux: les échanges commerciaux, les investissements directs étrangers (IDE), l'aide publique et les transferts des migrants. Nos estimations suggèrent que les IDE augmentent les inégalités, à l'opposé des transferts des migrants. L'ouverture semble être favorable aux populations pauvres dans les pays à revenu intermédiaire, tandis que l'aide est favorable aux classes moyennes dans les pays démocratiques. Des simulations suggèrent qu'en moyenne le fort impact négatif des IDE sur les inégalités n'est pas totalement compensé par l'influence plutôt égalitaire des trois autres sources de financement. De plus, les pays africains semblent se distinguer en ce qui concerne l'impact distributif des flux commerciaux et d'aide.

Mots-clefs : Inégalités, aide, transferts des migrants, IDE, ouverture commerciale

\begin{abstract}
We propose an econometric analysis of the distributive impact of trade flows, foreign direct investment (FDI), official aid and migrants' remittances. Results suggest that FDI increases inequality, while remittances tend to reduce inequality. Trade and aid have a non-linear relationship with income distribution: trade favours the poorest in middle income countries while aid favours the middle class in democracies. Simulations suggest that, on average, the highly adverse impact on distribution of FDI is not compensated by the other three sources of financing. Moreover, African countries show a different pattern of distributional impact of trade and aid.
\end{abstract}

Key Words: Inequality, aid, remittances, FDI, trade openness

JEL Code : D31, F2, F35 


\section{Table des matières}

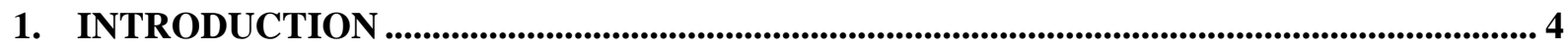

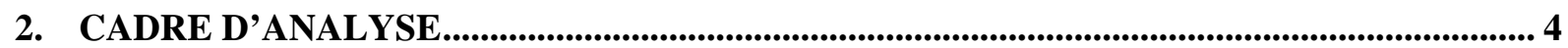

3. MODELE ECONOMETRIQUE, DONNEES ET METHODOLOGIE..................................... 5

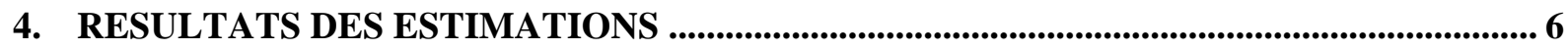

4.1. Des non-linéarités dans la relation ouverture commerciale - inégalité ..................................... 7

4.2. L'aide favorise les classes moyennes dans les pays démocratiques............................................ 7

4.3. Analyse comparée de l’impact distributif des différents flux de financement .......................... 10

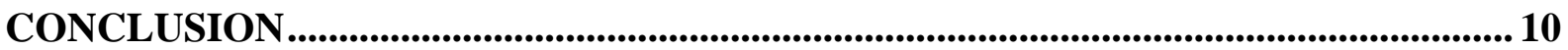

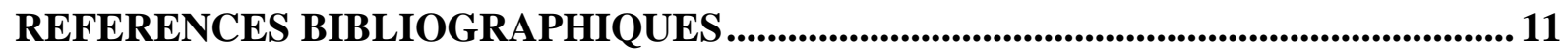

\section{Liste des tableaux}

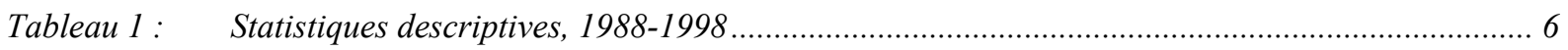

Tableau 2 : $\quad$ Des non-linéarités dans la relation inégalité-financement international ?............................... 7

Tableau 3 : $\quad$ Financement du développement et inégalités intra-pays, 1988-1998, 64 pays......................... 9

Tableau 4 : $\quad$ Simulations de l'influence des flux internationaux sur les inégalités internes ${ }^{l}$......................... 10

Tableau A 1 : Estimation de l'équation (3), spécification de base, 1988-1998, 64 pays............................... 13

Tableau A 2: Influence des flux de financement en fonction du niveau de développement, 1988-1998,

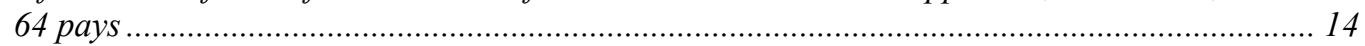

Tableau A 3 : Influence des flux de financement en fonction du caractère démocratique, 1988-1998,

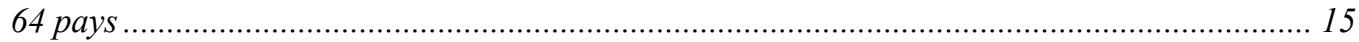

Tableau A 4 : Existe-t-il une spécificité africaine dans la relation aide-inégalités ? 1988-1998, 64 pays........ 16 


\section{INTRODUCTION}

Dans l'intense débat sur l'évolution des inégalités dans le monde, il existe au moins un consensus : les inégalités internes de revenu ont augmenté dans les pays en voie de développement depuis le milieu des années 80 (Banque mondiale [2006]). L'influence de la mondialisation ${ }^{1}$ sur cet état de fait porte en revanche à controverse : certaines études suggèrent que l'ouverture aux échanges internationaux est sans effet sur les inégalités ${ }^{2}$; d'autres, au contraire, défendent qu'elle augmente les inégalités dans les pays pauvres ${ }^{3}$. Toutefois, aucune étude n'a permis de mettre en évidence un effet égalitaire de l'ouverture dans les pays en développement. De même, les investissements directs étrangers (IDE) ne semblent pas favorables aux plus pauvres.

Malgré des effets très contrastés sur la distribution des revenus dans les pays en développement, les flux commerciaux et les IDE sont appelés à la rescousse de l'aide dans le financement du développement. Ainsi, la conférence de Monterrey en 2002, établit-elle que la réalisation des Objectifs du Millénaire pour le Développement nécessite un doublement des flux d'aide et un rôle accru des flux commerciaux et des IDE dans le financement du développement. Les transferts des migrants sont également vus, notamment par les détracteurs de l'aide, comme une source importante de financement du développement (Rajan et Subramanian [2005]).

Dans cet article, nous proposons une évaluation comparée de l'influence sur les inégalités internes des pays en développement de ces différentes sources de financement international : flux commerciaux, investissements directs étrangers, aide publique au développement et transferts privés des migrants. Adopter une approche intra-pays est original, puisque les rares études visant à comparer l'influence de ces flux sur les inégalités ont plutôt opté pour une approche inter-pays (Cogneau et Lambert [2006] ; Bourguignon et al. [2006]). Nous présentons tout d'abord notre cadre d'analyse, puis le modèle économétrique que nous estimons. Enfin, nous discutons les résultats économétriques.

\section{CADRE D'ANALYSE}

Suivant les travaux économétriques qui explorent la relation entre les échanges internationaux et les inégalités de revenu, nous optons pour l'estimation d'un modèle en forme réduite. Ainsi, ce modèle capte-t-il l'impact agrégé d'un ensemble de mécanismes par lesquels les différents flux internationaux peuvent influencer la distribution des revenus.

Ces mécanismes aboutissent à des effets a priori ambigus de nos variables d'intérêt sur les inégalités. L'impact distributif d'une plus grande ouverture aux échanges internationaux est la combinaison d'effets d'équilibre général sur les revenus des facteurs et sur les prix des biens. Ces effets dépendent des dotations relatives des pays en facteurs de production, du cadre plus ou moins rigide des différents marchés internes et de la manière dont les revenus des facteurs sont alloués au sein de l'ensemble de la population.

Outre ces déterminants, l'impact distributif des investissements directs étrangers dépend aussi des effets d'entraînement que peuvent susciter les entreprises multinationales dans les pays en développement, ainsi que des politiques de rapatriement des bénéfices. Les mécanismes qui régissent l'impact distributif des migrations internationales ne sont pas réellement différents de ceux qui déterminent l'effet des IDE. Les migrations internationales modifient les allocations en emplois qualifiés et non-qualifiés d'un pays, et donc les rémunérations relatives des facteurs et les prix relatifs des biens ; les transferts monétaires des migrants internationaux exercent, quant à eux, un effet revenu sur les récipiendaires dont l’impact sur les inégalités est a priori indéterminé.

\footnotetext{
Le phénomène de mondialisation est généralement défini à partir d’indicateurs de résultats - par exemple un accroissement de la part du commerce dans le PIB et une augmentation des flux d'investissements directs étrangers.

Voir Birdsall et Londono [1998], Li et al. [1998] ou Dollar et Kraay [2002].

Voir Barro [2000], Ravallion [2001] ou Milanovic [2005].

Les travaux ne trouvent pas d'effet significatif sur les inégalités (Vivarelli [2004] ; Milanovic [2005]), ou montrent que les IDE tendent à accroître la dispersion des rémunérations (Velde Te \& Morrissey [2001, 2002] ; Rama [2003]).
} 
L'influence de l'aide internationale sur les inégalités peut être plus ou moins directe et dépend de sa finalité. Ses canaux de transmission sont différents si elle est allouée à des projets d'infrastructures ou à des projets d'éducation et de santé, ou encore si elle est distribuée directement auprès des populations. Son impact distributif dépend aussi des priorités des pays donateurs et récipiendaires. Enfin, quelle que soit la finalité de l'aide, le mode de fonctionnement des institutions dans les pays en développement est susceptible d'influencer son effet distributif.

Si la plupart des travaux économétriques explorant l'impact de l'ouverture commerciale et des IDE sur la distribution des revenus adoptent une approche en forme réduite, les mesures des inégalités utilisées varient d'une étude à l'autre : certaines cherchent à expliquer l'évolution des distributions de salaires ; d'autres utilisent les bases internationales d'indicateurs d'inégalité sur un large échantillon de pays les bases de Deininger et Squire [1997] ou de WIDER [2005], par exemple. A l'instar de Milanovic [2005], nous exploitons la base World Income Distribution ${ }^{5}$ de la Banque mondiale. Cette base est une compilation de données primaires d'enquêtes auprès des ménages sur les déciles de niveau de vie. Elle est donc de meilleure qualité que les bases généralement utilisées et couvre un large panel de pays sur trois périodes temporelles (1988, 1993 et 1998). Fait original, elle permet d'étudier l'impact distributif décile par décile des différents flux de financement international ${ }^{6}$.

\section{MODELE ECONOMETRIQUE, DONNEES ET METHODOLOGIE}

Le modèle économétrique que nous estimons est proche de celui proposé par Milanovic [2005]. Le niveau de revenu absolu du décile $i$ dans le pays $j$, en $t$, peut s'écrire comme une fonction d'un indicateur d’inégalité $\left(I_{j t}\right)$ et du revenu moyen du pays $\left(m_{j t}\right)$ :

$$
y_{i j t}=f\left(I_{j t}, m_{j t}\right) \text {. }
$$

Ainsi, le revenu relatif du décile $i$ peut s'écrire :

$$
y_{i j t} / m_{j t}=g\left(I_{j t}\right)
$$

Les inégalités, $I_{j t}$, sont saisies par un ensemble de facteurs. Nous choisissons de capter les facteurs structurels des inégalités de manière agrégée par le PIB moyen par habitant des pays ${ }^{7}$. Pour saisir la relation non-linéaire entre le niveau de développement et les inégalités (relation de Kuznets), nous introduisons également le revenu au carré. L’indicateur d'inégalité est donc supposé dépendre du revenu moyen par habitant et des différents éléments participant de la mondialisation (ouverture commerciale, IDE, aide, transferts des migrants). L’équation estimée est de la forme :

$$
\begin{aligned}
& y_{i j t} / m_{j t}=\alpha_{i 1}+\alpha_{i 2} \text { Revenu }_{j t}+\alpha_{i 3} \text { Revenu }_{j t}{ }^{2}+\alpha_{i 4} \text { Ouverture }_{j t}+\alpha_{i 5} I D E_{j t} \\
& +\alpha_{i 6} \text { Aide }_{j t}+\alpha_{i 7} \text { Transfert }_{j t}+u_{i j t}
\end{aligned}
$$

Le revenu par habitant est en parité de pouvoir d'achat (Penn World Tables 6.1), en dollars constants. La variable d'ouverture qui nous intéresse est l'ouverture commerciale observée (exportations plus importations rapportées au PIB). Les investissements directs étrangers sont extraits de la base de données de Milanovic [2005] et sont calculés à partir des données de la CNUCED. L’aide est saisie par les déboursements nets d'aide publique au développement tels que définis par le Comité d'aide au développement de l'OCDE. Enfin, les transferts des migrants sont issus des World Development Indicators [2004]. Les variables d'ouverture, d'IDE, d'aide et de transferts sont toutes mesurées en pourcentage du PIB. Les déciles de revenus sont disponibles pour trois années (1988, 1993 et 1998) et les variables de droite ont été mesurées en moyenne sur les sous-périodes 1984-1988, 1989-1993 et 1994-1998.

Nous remercions Branko Milanovic de nous avoir fourni cette base de données.

Afin de disposer d'un échantillon suffisamment important nous avons ajouté 9 observations extraites de la base WIDER pour lesquelles la mesure des déciles de revenu est identique à celle de World Income Distribution et l'indicateur de qualité est égal à 1 ou 2 (WIDER [2005]).

Milanovic [2005] saisit les facteurs structurels par le niveau moyen de consommation des ménages, qui nous semble moins saisir l'ensemble des effets de structure que le PIB par habitant. L'introduction de variables participant au développement (stock de capital humain, profondeur financière, dualisme agricole, etc.) n’a pas été possible car l'échantillon de pays pour lesquels ces informations sont disponibles aurait alors été trop réduit. Voir Bourguignon et Morrisson, [1998] et Li et al. [1998] pour une discussion sur l'impact de ces facteurs. 
Tableau 1 : Statistiques descriptives, 1988-1998

\begin{tabular}{lccccccccc}
\hline & & Pays & Gini $^{1}$ & PIB $^{2}$ & Démo. $^{3}$ & Ouv. $^{4}$ & IDE $^{4}$ & Aide $^{4}$ & Transf. $^{4}$ \\
\hline Afrique & 1988 & 16 & 42,3 & 2105 & 1 & 65 & 0,8 & 9,8 & 1,2 \\
& 1998 & 20 & 44,1 & 1694 & 3 & 71 & 2,0 & 11,7 & 1,3 \\
\hline Asie & 1988 & 11 & 36,7 & 3310 & 5 & 44 & 0,5 & 2,0 & 1,7 \\
& 1998 & 15 & 38,3 & 4360 & 12 & 69 & 2,0 & 2,7 & 1,3 \\
\hline Am. latine & 1988 & 17 & 47,8 & 4838 & 13 & 47 & 0,8 & 2,7 & 0,7 \\
& 1998 & 18 & 51,3 & 6089 & 17 & 69 & 3,4 & 3,2 & 1,6 \\
\hline Total & 1988 & 44 & 43,1 & 3462 & 19 & 53 & 0,7 & 5,1 & 1,1 \\
& 1998 & 53 & 44,9 & 3942 & 32 & 70 & 2,5 & 6,3 & 1,4 \\
\hline
\end{tabular}

1) indicateur moyen des inégalités internes, par continent, à partir du calcul des Gini de chaque pays, sources : calculs des auteurs à partir de Milanovic [2005] ; 2) en dollars PPA à prix constants 1996 per capita, source: Pen World Table 6.1; 3) nombre de pays démocratiques : définis comme les pays ayant l'indicateur de démocratie de Polity IV supérieur à la médiane de notre échantillon (4,8 sur une échelle de 0 à 10) ; 4) en pourcentage du PIB et en moyenne sur les périodes 1984-88 \& 1994-98, sources : WDI [2004], Milanovic [2005] et OCDE-CAD.

Le Tableau 1 met en évidence l'augmentation des inégalités entre 1988 et 1998, de 2 points de Gini en moyenne. Parallèlement, l'ouverture commerciale, les IDE, l'aide et les transferts des migrants ont également augmenté en moyenne. L’Afrique paraît toutefois se distinguer de l'Asie et de l'Amérique latine : le continent africain dépend de façon importante de l'aide publique ; les IDE et les transferts de migrants y ont, en moyenne, relativement moins augmenté que dans les autres pays en développement. De plus, si le nombre de pays démocratiques a partout augmenté entre 1988 et 1998, leur proportion demeure très faible en Afrique.

Deux sources de biais d'endogénéité peuvent être particulièrement importantes dans l'estimation de l'équation (3). Tout d'abord, une relation de double causalité est susceptible d'exister entre certaines variables de droite (le revenu, notamment) et la part des revenus des différents déciles dans le revenu moyen. Ensuite, l'omission de variables corrélées à la fois avec les inégalités et avec les variables explicatives est également une source importante d'endogénéité ${ }^{8}$. Nous instrumentons donc l'ensemble des variables de droite et estimons le modèle par la méthode des moments généralisés. Des variables muettes régionales sont également introduites pour saisir une source régionale d'hétérogénéité inobservable ${ }^{9}$ - les pays africains et d'Amérique latine ont, toutes choses égales par ailleurs, des distributions de revenus plus inégalitaires que les pays asiatiques. Les instruments utilisés sont l'ensemble des variables endogènes retardées d'une période, ainsi que la population retardée d'une période, et des variables de distance géographique et culturelle, introduites linéairement et en interaction avec les montants totaux d'aide des cinq principaux pays donneurs (Tavares [2003]) ${ }^{10}$.

\section{RESULTATS DES ESTIMATIONS}

Les conclusions des estimations de l'équation (3), décile par décile, sont résumées dans la première colonne du Tableau 2 et sont présentées dans le Tableau A1 en Annexe. Les pays de notre échantillon se situent sur la partie ascendante de la courbe de Kuznets, les inégalités augmentant avec le niveau de développement mais de manière décroissante. L'ouverture et l'aide ne semblent pas influencer significativement les inégalités, tandis que les IDE et les transferts des migrants ont respectivement une influence défavorable et favorable aux plus pauvres.

\footnotetext{
Les chocs externes, par exemple, sont susceptibles d'augmenter les inégalités et d’être également corrélés avec l'aide ou l’ouverture commerciale.

9 Des effets spécifiques pays pourraient également être introduits pour tenir compte de l’hétérogénéité inobservable des pays. Mais la profondeur temporelle de notre panel est faible (d’autant plus que l’année 1988 est moins bien renseignée que les années 1993 et 1998 ) et l'estimateur within donne de mauvais résultats.

10 La validité des instruments est testée par un test de Hansen de sur-identification.
} 


\subsection{Des non-linéarités dans la relation ouverture commerciale - inégalité}

L'influence des flux de financements internationaux peut dépendre du niveau de développement. Par exemple, les analyses de l'impact distributif de l'ouverture commerciale montrent que l'ouverture tend plutôt à être égalitaire dans les pays « riches » et inégalitaire dans les pays « pauvres » ${ }^{11}$.

A partir de l'estimation de l'équation (3), nous tentons donc d'identifier si l'influence de l'ouverture commerciale, des IDE, de l'aide et des transferts des migrants dépend du niveau de développement. Ces quatre variables sont introduites en interaction avec le revenu par habitant. La colonne (2) du Tableau 2 résume les résultats de ces estimations qui sont présentées dans leur intégralité dans le Tableau A2 en Annexe.

Conformément à l'hypothèse de non-linéarité, l’ouverture commerciale semble avoir une influence favorable sur les revenus des plus pauvres dans les pays ayant atteint un certain niveau de développement ${ }^{12}$. Le cas des IDE est plus complexe. Choisir entre la relation linéaire de la colonne (1) et la relation non-linéaire de la colonne (2) est en effet délicat, puisque l'influence significative de l'interaction des IDE avec le revenu se traduit par la perte de significativité de la variable d'IDE. Le point intéressant est toutefois que, linéaire ou non, la relation entre les IDE et la distribution des revenus semble très défavorable aux plus pauvres. Enfin, la colonne (2) suggère que l'influence de l'aide et des transferts des migrants ne dépend pas du niveau de développement.

Tableau 2 : Des non-linéarités dans la relation inégalité-financement international ?

\begin{tabular}{|c|c|c|c|c|}
\hline \multicolumn{2}{|c|}{ Spécification } & \multicolumn{3}{|c|}{ Effet sur les inégalités } \\
\hline & & (1) & $(2)$ & (3) \\
\hline \multirow[t]{6}{*}{ Equation (3) } & Revenu p.c. & + & n.s & n.s \\
\hline & Revenu p.c., au carré & - & n.s & n.s \\
\hline & Ouverture & n.s & + & + \\
\hline & IDE & + & n.s & n.s \\
\hline & Aide & n.s & n.s & + \\
\hline & Transferts & - & n.s & - \\
\hline \multirow[t]{4}{*}{ Fonction du revenu p.c. } & Ouverture x revenu p.c. & & - & - \\
\hline & IDE x revenu p.c. & & + & \\
\hline & Aide x revenu p.c. & & n.s & \\
\hline & Transferts x revenu p.c. & & n.s & \\
\hline \multirow[t]{3}{*}{ Fonction des institutions } & IDE $\mathrm{x}$ démocratie & & & + \\
\hline & Aide x démocratie & & & - \\
\hline & Transferts x démocratie & & & n.s \\
\hline
\end{tabular}

n.s : non significatif. Résume les conclusions des tests de spécifications mis en œuvre à partir de l'estimation de l'équation (3) par déciles. Les tableaux de régressions sont présentés dans Chauvet et Mesplé-Somps [2007]. Pour estimer la spécification de la colonne (3), une variable muette « démocratie » a également été introduite dans les estimations.

\subsection{L’aide favorise les classes moyennes dans les pays démocratiques}

L'absence d'effet de l'aide sur la distribution des revenus, suggérée par les colonnes 1 et 2 du Tableau 2, peut s'expliquer par un autre type de non-linéarités dans la relation aide - inégalité : celles liées à la qualité du fonctionnement institutionnel des pays receveurs. L’impact distributif de l'aide publique au développement peut être influencé par la capacité des institutions à : (i) assurer ou non une bonne gestion des finances publiques; (ii) responsabiliser les élus vis-à-vis des électeurs ('accountability'). C’est essentiellement ce deuxième effet auquel nous nous intéressons.

Dans le Tableau 3, nous introduisons donc une variable muette " démocratie » et son interaction avec l'aide au développement. Nous avons procédé à des tests de cette spécification en nous assurant que l'introduction de la variable démocratie en interaction avec les IDE et les transferts des migrants ne modifie pas les résultats (voir la colonne (3) du Tableau 2 et le Tableau A3 en Annexe).

11 Voir Anderson [2005] pour une revue de littérature.

2 Le point de retournement correspond au revenu par habitant du Brésil. 
Le Tableau 3 confirme la relation non-linéaire de l'ouverture commerciale avec la distribution des revenus. Dans les pays ayant un revenu par habitant supérieur à celui de la Tunisie (5.800 \$PPA), l'ouverture commerciale favorise les parts de revenu des plus pauvres et diminue celles des plus riches. Pour les autres pays, l'ouverture commerciale exerce un impact distributif inverse ${ }^{13}$.

En nous fondant sur le Tableau 2, nous avons opté pour une relation linéaire entre les IDE et la distribution des revenus. Mais introduire les non-linéarités ne modifie pas les résultats concernant les autres variables. Les IDE ont une influence très fortement défavorable sur les parts de revenu des populations pauvres.

L’influence de l'aide semble dépendre du caractère démocratique ou non des pays en développement. Dans les pays non-démocratiques, l'aide est défavorable aux plus pauvres et aux classes moyennes (déciles 1 à 6) et favorable aux déciles 9 et 10. Dans les pays démocratiques, l'effet de l'aide demeure légèrement négatif pour les populations très pauvres (déciles 1 à 3 ). Elle est en revanche favorable aux classes moyennes (déciles 4 à 7) et est défavorable aux plus riches (décile 10) ${ }^{14}$.

Enfin, les transferts des migrants semblent fortement réduire les inégalités et ce, indépendamment du niveau de développement et de la qualité des institutions.

3 Tel est le cas de l'ensemble des pays d'Afrique Sub-saharienne de notre échantillon.

La majorité des pays africains présents dans notre échantillon ne sont pas démocratiques (voir Tableau 1). Nous avons donc exploré la possibilité que la non-linéarité entre l'aide et la muette « démocratie » saisisse en fait une spécificité africaine. L’introduction d’une variable interactive de l'aide avec la muette africaine n'est cependant jamais significative (voir Tableau A4 en Annexe). 
Tableau 3 : Financement du développement et inégalités intra-pays, 1988-1998, 64 pays.

\begin{tabular}{|c|c|c|c|c|c|c|c|c|c|c|}
\hline & $\begin{array}{c}1^{\mathrm{er}} \text { décile } \\
(1)\end{array}$ & $\begin{array}{c}2^{\text {ème }} \text { décile } \\
(2)\end{array}$ & $\begin{array}{c}3^{\text {ème }} \text { décile } \\
(3)\end{array}$ & $\begin{array}{c}4^{\text {ème }} \text { décile } \\
(4)\end{array}$ & $\begin{array}{c}5^{\text {ème }} \text { décile } \\
\text { (5) }\end{array}$ & $\begin{array}{c}6^{\text {ème }} \text { décile } \\
(6)\end{array}$ & $\begin{array}{c}7^{\text {ème }} \text { décile } \\
(7)\end{array}$ & $\begin{array}{c}8^{\text {ème }} \text { décile } \\
(8)\end{array}$ & $\begin{array}{c}9^{\text {ème }} \text { décile } \\
(9)\end{array}$ & $\begin{array}{c}10^{\text {ème }} \text { décile } \\
\text { (10) }\end{array}$ \\
\hline Ouverture (\% PIB) & $\begin{array}{c}-0.070 * \\
(1.74)\end{array}$ & $\begin{array}{c}-0.096 * \\
(1.94)\end{array}$ & $\begin{array}{c}-0.095^{*} \\
(1.92)\end{array}$ & $\begin{array}{c}-0.099 * * \\
(2.05)\end{array}$ & $\begin{array}{c}-0.094 * * \\
(1.97)\end{array}$ & $\begin{array}{l}-0.064 \\
(1.34)\end{array}$ & $\begin{array}{l}-0.041 \\
(0.87)\end{array}$ & $\begin{array}{c}0.044 \\
(1.00)\end{array}$ & $\begin{array}{c}0.093 * \\
(1.71)\end{array}$ & $\begin{array}{c}0.532 \\
(1.60)\end{array}$ \\
\hline Ouverture x Revenu p.c. & $\begin{array}{c}0.010^{*} \\
(1.67)\end{array}$ & $\begin{array}{c}0.014 * * \\
(2.01)\end{array}$ & $\begin{array}{c}0.014^{* *} \\
(1.99)\end{array}$ & $\begin{array}{c}0.015^{* *} \\
(2.24)\end{array}$ & $\begin{array}{c}0.015^{* *} \\
(2.18)\end{array}$ & $\begin{array}{l}0.011 \\
(1.63)\end{array}$ & $\begin{array}{l}0.007 \\
(1.08)\end{array}$ & $\begin{array}{l}-0.004 \\
(0.57)\end{array}$ & $\begin{array}{c}-0.014 * \\
(1.70)\end{array}$ & $\begin{array}{c}-0.085 * \\
(1.83)\end{array}$ \\
\hline IDE (\% PIB) & $\begin{array}{c}-1.586^{* *} \\
(2.40)\end{array}$ & $\begin{array}{c}-1.661 * * \\
(2.06)\end{array}$ & $\begin{array}{c}-1.885^{* *} \\
(2.19)\end{array}$ & $\begin{array}{c}-2.172^{* *} \\
(2.45)\end{array}$ & $\begin{array}{c}-2.127 * * \\
(2.39)\end{array}$ & $\begin{array}{c}-2.033^{* *} \\
(2.30)\end{array}$ & $\begin{array}{c}-1.481^{*} \\
(1.72)\end{array}$ & $\begin{array}{l}-0.741 \\
(0.88)\end{array}$ & $\begin{array}{l}1.510 \\
(1.41)\end{array}$ & $\begin{array}{c}12.745^{* *} \\
(1.97)\end{array}$ \\
\hline Aide (\% PIB) & $\begin{array}{c}-0.512^{* *} \\
(2.34)\end{array}$ & $\begin{array}{c}-0.509 * \\
(1.95)\end{array}$ & $\begin{array}{c}-0.603 * * \\
(2.13)\end{array}$ & $\begin{array}{c}-0.723^{* *} \\
(2.37)\end{array}$ & $\begin{array}{c}-0.682^{* *} \\
(2.20)\end{array}$ & $\begin{array}{c}-0.574 * \\
(1.81)\end{array}$ & $\begin{array}{l}-0.271 \\
(0.89)\end{array}$ & $\begin{array}{l}0.061 \\
(0.24)\end{array}$ & $\begin{array}{l}0.575 * \\
(1.87)\end{array}$ & $\begin{array}{c}3.453^{*} \\
(1.65)\end{array}$ \\
\hline Aide x Muette Démocratie & $\begin{array}{l}0.452^{*} \\
(1.96)\end{array}$ & $\begin{array}{l}0.469^{*} \\
(1.87)\end{array}$ & $\begin{array}{c}0.597 * * \\
(2.23)\end{array}$ & $\begin{array}{c}0.772 * * * \\
(2.65)\end{array}$ & $\begin{array}{c}0.773 * * * \\
(2.60)\end{array}$ & $\begin{array}{c}0.669 * * \\
(2.20)\end{array}$ & $\begin{array}{l}0.387 \\
(1.33)\end{array}$ & $\begin{array}{l}-0.034 \\
(0.14)\end{array}$ & $\begin{array}{c}-0.555^{*} \\
(1.83)\end{array}$ & $\begin{array}{c}-3.819 * \\
(1.91)\end{array}$ \\
\hline Muette démocratie & $\begin{array}{r}-0.000 \\
(0.01)\end{array}$ & $\begin{array}{l}0.009 \\
(0.30)\end{array}$ & $\begin{array}{l}0.005 \\
(0.16)\end{array}$ & $\begin{array}{l}0.005 \\
(0.14)\end{array}$ & $\begin{array}{l}-0.001 \\
(0.02)\end{array}$ & $\begin{array}{l}-0.010 \\
(0.27)\end{array}$ & $\begin{array}{l}-0.020 \\
(0.56)\end{array}$ & $\begin{array}{l}-0.034 \\
(0.89)\end{array}$ & $\begin{array}{l}-0.045 \\
(0.86)\end{array}$ & $\begin{array}{l}0.065 \\
(0.25)\end{array}$ \\
\hline Transferts des migrants (\% PIB) & $\begin{array}{c}0.920 * * * \\
(3.46)\end{array}$ & $\begin{array}{c}1.068 * * * \\
(3.52)\end{array}$ & $\begin{array}{c}0.975^{* * *} \\
(3.17)\end{array}$ & $\begin{array}{c}0.903 * * * \\
(2.92)\end{array}$ & $\begin{array}{c}0.836 * * * \\
(2.97)\end{array}$ & $\begin{array}{c}0.838 * * * \\
(2.91)\end{array}$ & $\begin{array}{l}0.607 * \\
(1.75)\end{array}$ & $\begin{array}{l}0.129 \\
(0.33)\end{array}$ & $\begin{array}{l}-0.341 \\
(0.64)\end{array}$ & $\begin{array}{c}-7.310 * * * \\
(3.22)\end{array}$ \\
\hline Revenu p.c. & $\begin{array}{c}-0.030 * * * \\
(4.06)\end{array}$ & $\begin{array}{c}-0.029 * * * \\
(3.54)\end{array}$ & $\begin{array}{c}-0.031 * * * \\
(3.32)\end{array}$ & $\begin{array}{c}-0.033^{* * *} \\
(3.09)\end{array}$ & $\begin{array}{c}-0.029 * * * \\
(2.63)\end{array}$ & $\begin{array}{c}-0.022 * \\
(1.87)\end{array}$ & $\begin{array}{l}-0.007 \\
(0.61)\end{array}$ & $\begin{array}{l}0.008 \\
(0.73)\end{array}$ & $\begin{array}{c}0.049 * * * \\
(3.51)\end{array}$ & $\begin{array}{c}0.127 * \\
(1.69)\end{array}$ \\
\hline Revenu p.c., carré & $\begin{array}{c}0.002^{* * *} \\
(2.84)\end{array}$ & $\begin{array}{c}0.001^{* *} \\
(2.24)\end{array}$ & $\begin{array}{c}0.002^{* *} \\
(2.46)\end{array}$ & $\begin{array}{c}0.002 * * \\
(2.33)\end{array}$ & $\begin{array}{c}0.002 * * \\
(2.01)\end{array}$ & $\begin{array}{l}0.001 \\
(1.64)\end{array}$ & $\begin{array}{l}0.001 \\
(0.74)\end{array}$ & $\begin{array}{l}0.000 \\
(0.08)\end{array}$ & $\begin{array}{c}-0.002^{* *} \\
(2.46)\end{array}$ & $\begin{array}{l}-0.008 \\
(1.32)\end{array}$ \\
\hline Muette Afrique & $\begin{array}{c}-0.043^{*} \\
(1.73)\end{array}$ & $\begin{array}{l}-0.044 \\
(1.47)\end{array}$ & $\begin{array}{l}-0.038 \\
(1.16)\end{array}$ & $\begin{array}{l}-0.022 \\
(0.64)\end{array}$ & $\begin{array}{l}-0.014 \\
(0.40)\end{array}$ & $\begin{array}{l}-0.009 \\
(0.24)\end{array}$ & $\begin{array}{l}-0.005 \\
(0.13)\end{array}$ & $\begin{array}{l}-0.013 \\
(0.36)\end{array}$ & $\begin{array}{l}0.002 \\
(0.06)\end{array}$ & $\begin{array}{l}0.092 \\
(0.37)\end{array}$ \\
\hline Muette Amérique latine & $\begin{array}{c}-0.133^{* * *} \\
(8.56)\end{array}$ & $\begin{array}{c}-0.138 * * * \\
(7.85)\end{array}$ & $\begin{array}{c}-0.126^{* * *} \\
(6.79)\end{array}$ & $\begin{array}{c}-0.111^{* * *} \\
(5.77)\end{array}$ & $\begin{array}{c}-0.094^{* * *} \\
(4.91)\end{array}$ & $\begin{array}{c}-0.078 * * * \\
(4.13)\end{array}$ & $\begin{array}{c}-0.051^{* * *} \\
(2.94)\end{array}$ & $\begin{array}{l}-0.010 \\
(0.60)\end{array}$ & $\begin{array}{c}0.075^{* * *} \\
(3.75)\end{array}$ & $\begin{array}{c}0.648 * * * \\
(5.01)\end{array}$ \\
\hline Constante & $\begin{array}{c}0.391^{* * *} \\
(16.67)\end{array}$ & $\begin{array}{c}0.513^{* * *} \\
(19.76)\end{array}$ & $\begin{array}{c}0.615^{* * *} \\
(20.87)\end{array}$ & $\begin{array}{c}0.712^{* * *} \\
(21.63)\end{array}$ & $\begin{array}{c}0.797 \\
(22.92)\end{array}$ & $\begin{array}{c}0.885 \\
(24.07)\end{array}$ & $\begin{array}{c}0.985 \\
(26.77)\end{array}$ & $\begin{array}{c}1.137 \\
(31.48)\end{array}$ & $\begin{array}{c}1.381 \\
(28.80)\end{array}$ & $\begin{array}{c}2.585 \\
(10.31)\end{array}$ \\
\hline Observations (pays) & $148(64)$ & $151(64)$ & $151(64)$ & $151(64)$ & $151(64)$ & $151(64)$ & $151(64)$ & $151(64)$ & $151(64)$ & $151(64)$ \\
\hline Hansen $\mathrm{J}$ test ( $p$-value) & 0.83 & 0.88 & 0.77 & 0.55 & 0.34 & 0.26 & 0.15 & 0.16 & 0.08 & 0.48 \\
\hline
\end{tabular}

Estimations par MMG (programme ivreg2.do développé par Baum et al. (2003)). Estimations robustes à l’hétéroscédasticité; * significatif à 10\%; ** à 5\%; *** à 1\%. 


\subsection{Analyse comparée de l'impact distributif des différents flux de financement}

Afin de comparer les effets sur les inégalités de chacune des différentes sources externes de financement du développement, nous procédons à des simulations sur la base des estimations du Tableau 3. Le Tableau 4 montre la contribution de chacun de ces flux sur les variations moyennes des inégalités des pays de l'échantillon entre 1988 et 1998. Il semble que l'ouverture commerciale a exercé une pression à la hausse des inégalités seulement dans les pays africains, pays à niveau faible de revenu, tandis que sur les autres continents elle a eu un effet plutôt égalitaire. Les IDE exercent un fort impact inégalitaire sur l'ensemble des continents. Les transferts internationaux des migrants ont permis de réduire les inégalités dans l'ensemble des pays, à l'exception des pays asiatiques. Enfin, l'impact égalitaire de l'aide ne semble jouer que dans les pays d'Asie et d'Amérique Latine. L'absence de systèmes démocratiques bien établis dans les pays africains de notre échantillon (voir Tableau 1) implique que l'aide n'y a pas eu un impact distributif favorable aux populations les plus démunies. Au total, il semble que les financements externes du développement ont eu un impact assez inégalitaire sur l'ensemble des continents.

Tableau 4 : Simulations de l’influence des flux internationaux sur les inégalités internes ${ }^{1}$

\begin{tabular}{lcccccc}
\hline & \multicolumn{2}{c}{$\begin{array}{c}\text { Variation moyenne } \\
\text { observée des inégalités }\end{array}$} & \multicolumn{2}{c}{ Variations moyennes simulées des inégalités } \\
\hline & & Ouverture & IDE & Aide & Transferts & Total \\
\hline & $(1)$ & $(2)$ & $(3)$ & $(4)$ & $(5)$ & $(6)$ \\
\hline Afrique & 1,9 & 0,4 & 2,3 & 0,1 & $-0,2$ & 2,7 \\
Asie & 0,4 & $-0,9$ & 1,9 & $-0,5$ & 0,1 & 0,6 \\
Amérique latine & 3,5 & $-0,1$ & 3,5 & $-0,1$ & $-0,5$ & 2,8 \\
\hline Echantillon total & 2,1 & $-0,2$ & 2,7 & $-0,2$ & $-0,2$ & 2,1 \\
\hline
\end{tabular}

1) Les variations sont exprimées en points de Gini. La première colonne est la variation moyenne observée des inégalités des pays pour lesquels les distributions de revenu sont disponibles en 1988 et 1998. Les colonnes 2 à 5 montrent la contribution de chacun des différents flux, et se lisent de la manière suivante : l'augmentation de l'ouverture commerciale entre 1988 et 1998 explique une augmentation de 0,4 point de Gini en moyenne dans les pays africains de l'échantillon (colonne 2). La colonne 6 synthétise les effets de l'ensemble des flux (colonnes 2 à 5).

\section{CONCLUSION}

Durant la décennie 1988-1998, l'augmentation des échanges commerciaux semble avoir eu, en moyenne, un impact relativement neutre sur les inégalités. A l'inverse, le triplement de la part des IDE dans le PIB des pays en développement a été un facteur d'aggravation assez important des inégalités. On ne peut alors espérer une contribution positive des IDE à la réalisation des OMD - notamment à la réduction de moitié de la pauvreté dans le monde d'ici à 2015 - qu'à condition qu'ils exercent un effet sur la croissance économique suffisamment élevé pour permettre une amélioration du niveau de vie de l'ensemble des populations. Les transferts des migrants exercent un effet compensatoire somme toute relativement modeste, tout comme l'aide, dont l'augmentation n'a pas suffi à renverser la tendance à la hausse des inégalités. 


\section{REFERENCES BIBLIOGRAPHIQUES}

Anderson E. (2005), « Openness and Inequality in Developing Countries: A Review of Theory and Recent Evidence », World Development, 33(7), p. 1045-1063.

Banque mondiale (2006), Equité et développement, Rapport sur le développement dans le monde 2006, Washington, D.C., The World Bank.

Barro R. (2000), « Inequality and Growth in a Panel if Countries », Journal of Economic Growth, 5(1), p. 5-32.

Baum C.F., Schaffer M.E., Stillman S. (2003), « Instrumental Variables and GMM: Estimation and Testing », The Stata Journal, 3(1), p. 1-31.

Birdsall N., Londono J-L. (1998), « No Trade-off: Efficient Growth via More Equal Human Capital Accumulation » in N. Birdsall, C. Graham et R. Sobot, eds, Beyond Tradeoffs: Market Reforms and Equitable Growth in Latin America, Washington, D.C., Inter-American Development Bank and Brooking Institution.

Bourguignon F., Morrisson C. (1998), « Inequality and Development: The Role of Dualism », Journal of Development Economics, 57(2), p. 233-258.

Bourguignon F., Levin V., Rosenblatt D. (2006), «Global Redistribution of Income », World Bank Policy Research Paper, 3961, July.

Chauvet L., Mesple-Somps S. (2006), « Impact des financements internationaux sur les inégalités des pays en développement », Document de travail DIAL. DT 2006-18, Paris.

Cogneau D., Lambert S. (2006), «Aide et cohérence des politiques du Nord », Document de travail Centre de Développement de l'OCDE, CEPREMAP, DIAL and LEA/INRA, 30 pp.

Deininger K., Squire L. (1997),, «A New Data Set Measuring Income Inequality », World Bank, Washington, D.C. (www.worldbank.org).

Dollar D., Kraay A. (2002), "Growth Is Good for the Poor », Journal of Economic Growth, 7(3), p. 195-225.

Li H., Squire L., Zou H. (1998), « Explaining International and Intertemporal Variations in Income Inequality », The Economic Journal, 108, p. 26-43.

Milanovic B. (2005),, «Can We Discern the Effect of Globalization on Income Distribution? Evidence from Household Surveys », The World Bank Economic Review, 19, p. 21-44.

Rajan R.G., Subramanian A. (2005), « What Undermines Aid’s Impact on Growth? », IMF Working Paper, 05/126.

Rama M. (2003), «Globalization and Workers in Developing Countries », World Bank Policy Research Working Paper, 2958, January, 38 p.

Ravallion M. (2001), "Growth, Inequality and Poverty: Looking beyond Averages », World Development, 29(11), p. 1803-15.

Tavares J. (2003), « Does Foreign Aid Corrupt? » Economics Letters, 79, p. 9-106.

Velde Te D. W., Morrissey O. (2001), « Foreign Ownership and Wages: Evidence from Five African Countries », CREDIT Research paper, 01/19, November, 37 p. 
Velde Te D. W., Morrissey O. (2002), « Foreign Direct Investment, Skills and Wage Inequality in East Asia », paper presented at the DESG conference in Nottingham, April 2002.

Vivarelli M. (2004), «Globalization, Skills and Within-Country Income Inequality in Developing Countries », in Lee E. and Vivarelli M. (ed.) Understanding Globalization, Employment and Poverty Reduction, Palgrave Macmillan, International Labour Office, London, 389 p.

WIDER (2005), World Income Inequality Database, V2.0a Use Guide and Data Sources. Database on www.wider.unu.edu 
Tableau A 1 : Estimation de l'équation (3), spécification de base, 1988-1998, 64 pays

\begin{tabular}{|c|c|c|c|c|c|c|c|c|c|c|}
\hline & $\begin{array}{c}1^{\mathrm{er}} \text { décile } \\
(1)\end{array}$ & $\begin{array}{c}2^{\text {ème }} \text { décile } \\
(2)\end{array}$ & $\begin{array}{c}3^{\text {ème }} \text { décile } \\
(3)\end{array}$ & $\begin{array}{c}4^{\text {ème }} \text { décile } \\
(4)\end{array}$ & $\begin{array}{c}5^{\text {ème }} \text { décile } \\
(5)\end{array}$ & $\begin{array}{c}6^{\text {ème }} \text { décile } \\
(6)\end{array}$ & $\begin{array}{c}7^{\text {ème }} \text { décile } \\
(7)\end{array}$ & $\begin{array}{c}8^{\text {ème }} \text { décile } \\
(8)\end{array}$ & $\begin{array}{c}9^{\text {ème }} \text { décile } \\
(9)\end{array}$ & $\begin{array}{c}10^{\text {ème }} \text { décile } \\
(10)\end{array}$ \\
\hline Ouverture (\% du PIB) & $\begin{array}{l}-0.028 \\
(1.06)\end{array}$ & $\begin{array}{l}-0.028 \\
(0.95)\end{array}$ & $\begin{array}{l}-0.032 \\
(1.03)\end{array}$ & $\begin{array}{l}-0.032 \\
(1.00)\end{array}$ & $\begin{array}{l}-0.032 \\
(1.01)\end{array}$ & $\begin{array}{l}-0.020 \\
(0.64)\end{array}$ & $\begin{array}{l}-0.009 \\
(0.30)\end{array}$ & $\begin{array}{l}0.032 \\
(1.23)\end{array}$ & $\begin{array}{l}0.044 \\
(1.23)\end{array}$ & $\begin{array}{l}0.150 \\
(0.68)\end{array}$ \\
\hline IDE (\% du PIB) & $\begin{array}{l}-1.28 \\
(2.22)^{* *}\end{array}$ & $\begin{array}{l}-1.270 \\
(1.92)^{*}\end{array}$ & $\begin{array}{l}-1.314 \\
(1.84)^{*}\end{array}$ & $\begin{array}{l}-1.401 \\
(1.86)^{*}\end{array}$ & $\begin{array}{l}-1.354 \\
(1.76)^{*}\end{array}$ & $\begin{array}{l}-1.446 \\
(1.85)^{*}\end{array}$ & $\begin{array}{l}-1.305 \\
(1.70)^{*}\end{array}$ & $\begin{array}{l}-1.221 \\
(1.60)\end{array}$ & $\begin{array}{l}0.231 \\
(0.25)\end{array}$ & $\begin{array}{l}9.879 \\
(1.75)^{*}\end{array}$ \\
\hline Aide (\% du PIB) & $\begin{array}{l}-0.276 \\
(1.84)^{*}\end{array}$ & $\begin{array}{l}-0.243 \\
(1.45)\end{array}$ & $\begin{array}{l}-0.235 \\
(1.33)\end{array}$ & $\begin{array}{l}-0.200 \\
(1.10)\end{array}$ & $\begin{array}{l}-0.130 \\
(0.73)\end{array}$ & $\begin{array}{l}-0.065 \\
(0.38)\end{array}$ & $\begin{array}{l}0.011 \\
(0.08)\end{array}$ & $\begin{array}{l}0.045 \\
(0.36)\end{array}$ & $\begin{array}{l}0.174 \\
(0.95)\end{array}$ & $\begin{array}{l}0.735 \\
(0.62)\end{array}$ \\
\hline Transferts (\% du PIB) & $\begin{array}{l}0.990 \\
(4.81)^{* * *}\end{array}$ & $\begin{array}{l}1.209 \\
(4.44)^{* * *}\end{array}$ & $\begin{array}{l}1.134 \\
(4.35)^{* * *}\end{array}$ & $\begin{array}{l}1.129 \\
(4.54)^{* * *}\end{array}$ & $\begin{array}{l}1.144 \\
(4.83)^{* * *}\end{array}$ & $\begin{array}{l}1.062 \\
(3.78)^{* * *}\end{array}$ & $\begin{array}{l}0.699 \\
(2.09)^{* *}\end{array}$ & $\begin{array}{l}0.072 \\
(0.19)\end{array}$ & $\begin{array}{l}-0.646 \\
(1.22)\end{array}$ & $\begin{array}{l}-8.290 \\
(3.62)^{* * *}\end{array}$ \\
\hline Revenu p.c. & $\begin{array}{l}-0.024 \\
(2.97)^{* * *}\end{array}$ & $\begin{array}{l}-0.024 \\
(2.62)^{* * *}\end{array}$ & $\begin{array}{l}-0.025 \\
(2.70)^{* * *}\end{array}$ & $\begin{array}{l}-0.023 \\
(2.41)^{* *}\end{array}$ & $\begin{array}{l}-0.018 \\
(1.77)^{*}\end{array}$ & $\begin{array}{l}-0.009 \\
(0.88)\end{array}$ & $\begin{array}{l}0.002 \\
(0.17)\end{array}$ & $\begin{array}{l}0.011 \\
(0.95)\end{array}$ & $\begin{array}{l}0.037 \\
(2.57)^{* *}\end{array}$ & $\begin{array}{l}0.059 \\
(0.82)\end{array}$ \\
\hline Revenu p.c. au carré & $\begin{array}{l}0.002 \\
(3.32)^{* * *}\end{array}$ & $\begin{array}{l}0.002 \\
(3.15)^{* * *}\end{array}$ & $\begin{array}{l}0.002 \\
(3.63)^{* * *}\end{array}$ & $\begin{array}{l}0.002 \\
(3.30)^{* * *}\end{array}$ & $\begin{array}{l}0.002 \\
(2.58)^{* * * *}\end{array}$ & $\begin{array}{l}0.001 \\
(1.64)\end{array}$ & $\begin{array}{l}0.000 \\
(0.54)\end{array}$ & $\begin{array}{l}-0.000 \\
(0.43)\end{array}$ & $\begin{array}{l}-0.002 \\
(2.55)^{* *}\end{array}$ & $\begin{array}{l}-0.007 \\
(1.58)\end{array}$ \\
\hline Muette Afrique & $\begin{array}{l}-0.068 \\
(4.99)^{* * *}\end{array}$ & $\begin{array}{l}-0.082 \\
(5.23)^{* * *}\end{array}$ & $\begin{array}{l}-0.078 \\
(4.79)^{* * *}\end{array}$ & $\begin{array}{l}-0.072 \\
(4.39)^{* * *}\end{array}$ & $\begin{array}{l}-0.058 \\
(3.59)^{* * *}\end{array}$ & $\begin{array}{l}-0.041 \\
(2.43)^{* *}\end{array}$ & $\begin{array}{l}-0.015 \\
(0.83)\end{array}$ & $\begin{array}{l}0.011 \\
(0.56)\end{array}$ & $\begin{array}{l}0.072 \\
(2.84)^{* * *}\end{array}$ & $\begin{array}{l}0.297 \\
(2.23)^{* *}\end{array}$ \\
\hline Muette Amérique latine & $\begin{array}{l}-0.137 \\
(8.85)^{* * *}\end{array}$ & $\begin{array}{l}-0.143 \\
(8.25)^{* * *}\end{array}$ & $\begin{array}{l}-0.132 \\
(7.34)^{* * *}\end{array}$ & $\begin{array}{l}-0.120 \\
(6.45)^{* * *}\end{array}$ & $\begin{array}{l}-0.104 \\
(5.66)^{* * *}\end{array}$ & $\begin{array}{l}-0.088 \\
(4.87)^{* * *}\end{array}$ & $\begin{array}{l}-0.062 \\
(3.72)^{* * *}\end{array}$ & $\begin{array}{l}-0.016 \\
(1.03)\end{array}$ & $\begin{array}{l}0.084 \\
(4.35)^{* * *}\end{array}$ & $\begin{array}{l}0.721 \\
(5.78)^{* * *}\end{array}$ \\
\hline Constant & $\begin{array}{l}0.368 \\
(17.72) * * *\end{array}$ & $\begin{array}{l}0.487 \\
(22.63) * * *\end{array}$ & $\begin{array}{l}0.584 \\
(25.15) * * *\end{array}$ & $\begin{array}{l}0.668 \\
(26.64) * * *\end{array}$ & $\begin{array}{l}0.745 \\
(28.19) * * *\end{array}$ & $\begin{array}{l}0.831 \\
(29.74) * * *\end{array}$ & $\begin{array}{l}0.942 \\
(31.84)^{* * *}\end{array}$ & $\begin{array}{l}1.117 \\
(35.49) * * *\end{array}$ & $\begin{array}{l}1.403 \\
(32.25) * * *\end{array}$ & $\begin{array}{l}2.909 \\
(13.80)^{* * * *}\end{array}$ \\
\hline Observations & 148 & 151 & 151 & 151 & 151 & 151 & 151 & 151 & 151 & 151 \\
\hline Hansen p-value & 0.60 & 0.79 & 0.72 & 0.55 & 0.39 & 0.36 & 0.25 & 0.24 & 0.24 & 0.54 \\
\hline
\end{tabular}

Estimations par MMG (programme ivreg2.do développé par Baum et al. (2003)). Estimations robustes à l’hétéroscédasticité; * significatif à 10\%; ** à 5\%; *** à 1\%. 
Tableau A 2 : Influence des flux de financement en fonction du niveau de développement, 1988-1998, 64 pays

\begin{tabular}{|c|c|c|c|c|c|c|c|c|c|c|}
\hline & $\begin{array}{l}1^{\mathrm{er}} \text { décile } \\
\text { (1) }\end{array}$ & $\begin{array}{c}2^{\text {ème }} \text { décile } \\
\text { (2) }\end{array}$ & $\begin{array}{l}3^{\text {ème }} \text { décile } \\
\text { (3) }\end{array}$ & $\begin{array}{l}4^{\text {ème }} \text { décile } \\
\text { (4) }\end{array}$ & $\begin{array}{c}5^{\text {ème }} \text { décile } \\
\text { (5) }\end{array}$ & $\begin{array}{l}6^{\text {ème }} \text { décile } \\
\text { (6) }\end{array}$ & $\begin{array}{c}7^{\text {ème }} \text { décile } \\
\text { (7) }\end{array}$ & $\begin{array}{c}8^{\text {ème }} \text { décile } \\
\text { (8) }\end{array}$ & $\begin{array}{l}9^{\text {ème }} \text { décile } \\
\text { (9) }\end{array}$ & $\begin{array}{c}10^{\text {ème }} \text { décile } \\
\text { (10) }\end{array}$ \\
\hline Ouverture (\% du PIB) & $\begin{array}{l}-0.117 \\
(2.38)^{* *}\end{array}$ & $\begin{array}{l}-0.175 \\
(2.69)^{* * *}\end{array}$ & $\begin{array}{l}-0.186 \\
(2.65)^{* * *}\end{array}$ & $\begin{array}{l}-0.197 \\
(2.70)^{* * *}\end{array}$ & $\begin{array}{l}-0.197 \\
(2.77)^{* * *}\end{array}$ & $\begin{array}{l}-0.173 \\
(2.41)^{* *}\end{array}$ & $\begin{array}{l}-0.122 \\
(1.78)^{*}\end{array}$ & $\begin{array}{l}0.013 \\
(0.20)\end{array}$ & $\begin{array}{l}0.184 \\
(2.76)^{* * *}\end{array}$ & $\begin{array}{l}1.137 \\
(2.32)^{* *}\end{array}$ \\
\hline Ouverture x Revenu p.c & $\begin{array}{l}0.019 \\
(2.17)^{* *}\end{array}$ & $\begin{array}{l}0.032 \\
(2.75)^{* * *}\end{array}$ & $\begin{array}{l}0.034 \\
(2.64)^{* * *}\end{array}$ & $\begin{array}{l}0.036 \\
(2.65)^{* * *}\end{array}$ & $\begin{array}{l}0.036 \\
(2.67)^{* * *}\end{array}$ & $\begin{array}{l}0.033 \\
(2.45)^{* *}\end{array}$ & $\begin{array}{l}0.026 \\
(2.01)^{* *}\end{array}$ & $\begin{array}{l}0.005 \\
(0.42)\end{array}$ & $\begin{array}{l}-0.030 \\
(2.35)^{* *}\end{array}$ & $\begin{array}{l}-0.216 \\
(2.41)^{* *}\end{array}$ \\
\hline IDE (\% du PIB) & $\begin{array}{l}-0.095 \\
(0.09)\end{array}$ & $\begin{array}{l}1.694 \\
(1.17)\end{array}$ & $\begin{array}{l}2.080 \\
(1.33)\end{array}$ & $\begin{array}{l}2.198 \\
(1.35)\end{array}$ & $\begin{array}{l}2.422 \\
(1.51)\end{array}$ & $\begin{array}{l}2.421 \\
(1.51)\end{array}$ & $\begin{array}{l}2.129 \\
(1.39)\end{array}$ & $\begin{array}{l}0.746 \\
(0.50)\end{array}$ & $\begin{array}{l}-0.673 \\
(0.39)\end{array}$ & $\begin{array}{l}-15.166 \\
(1.32)\end{array}$ \\
\hline IDE x Revenu p.c. & $\begin{array}{l}-0.310 \\
(1.14)\end{array}$ & $\begin{array}{l}-0.778 \\
(2.18)^{* *}\end{array}$ & $\begin{array}{l}-0.882 \\
(2.40)^{* *}\end{array}$ & $\begin{array}{l}-0.929 \\
(2.48)^{* *}\end{array}$ & $\begin{array}{l}-0.948 \\
(2.61)^{* * *}\end{array}$ & $\begin{array}{l}-0.932 \\
(2.70)^{* * *}\end{array}$ & $\begin{array}{l}-0.818 \\
(2.66)^{* * *}\end{array}$ & $\begin{array}{l}-0.448 \\
(1.57)\end{array}$ & $\begin{array}{l}0.254 \\
(0.63)\end{array}$ & $\begin{array}{l}6.197 \\
(2.57)^{* *}\end{array}$ \\
\hline Aide (\% du PIB) & $\begin{array}{l}-0.018 \\
(0.08)\end{array}$ & $\begin{array}{l}0.160 \\
(0.64)\end{array}$ & $\begin{array}{l}0.128 \\
(0.49)\end{array}$ & $\begin{array}{l}0.097 \\
(0.37)\end{array}$ & $\begin{array}{l}0.109 \\
(0.43)\end{array}$ & $\begin{array}{l}0.066 \\
(0.26)\end{array}$ & $\begin{array}{l}0.104 \\
(0.45)\end{array}$ & $\begin{array}{l}-0.059 \\
(0.26)\end{array}$ & $\begin{array}{l}-0.118 \\
(0.38)\end{array}$ & $\begin{array}{l}-0.797 \\
(0.45)\end{array}$ \\
\hline Aide x Revenu pc. & $\begin{array}{l}-0.080 \\
(0.61)\end{array}$ & $\begin{array}{l}-0.143 \\
(0.88)\end{array}$ & $\begin{array}{l}-0.112 \\
(0.64)\end{array}$ & $\begin{array}{l}-0.063 \\
(0.34)\end{array}$ & $\begin{array}{l}-0.041 \\
(0.22)\end{array}$ & $\begin{array}{l}0.003 \\
(0.02)\end{array}$ & $\begin{array}{l}-0.022 \\
(0.12)\end{array}$ & $\begin{array}{l}0.053 \\
(0.30)\end{array}$ & $\begin{array}{l}0.061 \\
(0.29)\end{array}$ & $\begin{array}{l}0.425 \\
(0.33)\end{array}$ \\
\hline Transferts (\% du PIB) & $\begin{array}{l}0.554 \\
(0.80)\end{array}$ & $\begin{array}{l}1.419 \\
(1.68)^{*}\end{array}$ & $\begin{array}{l}1.258 \\
(1.36)\end{array}$ & $\begin{array}{l}1.065 \\
(1.05)\end{array}$ & $\begin{array}{l}0.865 \\
(0.83)\end{array}$ & $\begin{array}{l}0.886 \\
(0.80)\end{array}$ & $\begin{array}{l}0.202 \\
(0.18)\end{array}$ & $\begin{array}{l}-0.714 \\
(0.76)\end{array}$ & $\begin{array}{l}-2.071 \\
(2.16)^{* *}\end{array}$ & $\begin{array}{l}-6.213 \\
(0.83)\end{array}$ \\
\hline Transferts x Revenu p.c. & $\begin{array}{l}0.124 \\
(0.60)\end{array}$ & $\begin{array}{l}-0.075 \\
(0.30)\end{array}$ & $\begin{array}{l}-0.057 \\
(0.20)\end{array}$ & $\begin{array}{l}-0.019 \\
(0.06)\end{array}$ & $\begin{array}{l}0.027 \\
(0.08)\end{array}$ & $\begin{array}{l}0.003 \\
(0.01)\end{array}$ & $\begin{array}{l}0.119 \\
(0.36)\end{array}$ & $\begin{array}{l}0.238 \\
(0.82)\end{array}$ & $\begin{array}{l}0.500 \\
(1.73)^{*}\end{array}$ & $\begin{array}{l}-0.563 \\
(0.24)\end{array}$ \\
\hline Revenu p.c. & $\begin{array}{l}-0.017 \\
(1.79) *\end{array}$ & $\begin{array}{l}-0.003 \\
(0.28)\end{array}$ & $\begin{array}{l}-0.002 \\
(0.18)\end{array}$ & $\begin{array}{l}-0.002 \\
(0.16)\end{array}$ & $\begin{array}{l}0.000 \\
(0.01)\end{array}$ & $\begin{array}{l}0.004 \\
(0.29)\end{array}$ & $\begin{array}{l}0.010 \\
(0.72)\end{array}$ & $\begin{array}{l}0.012 \\
(0.85)\end{array}$ & $\begin{array}{l}0.027 \\
(1.60)\end{array}$ & $\begin{array}{l}-0.058 \\
(0.59)\end{array}$ \\
\hline Revenu p.c. au carré & $\begin{array}{l}0.0004 \\
(0.45)\end{array}$ & $\begin{array}{l}-0.0006 \\
(0.58)\end{array}$ & $\begin{array}{l}-0.0005 \\
(0.45)\end{array}$ & $\begin{array}{l}-0.0005 \\
(0.39)\end{array}$ & $\begin{array}{l}-0.0005 \\
(0.44)\end{array}$ & $\begin{array}{l}-0.0006 \\
(0.45)\end{array}$ & $\begin{array}{l}-0.0008 \\
(0.68)\end{array}$ & $\begin{array}{l}-0.0004 \\
(0.37)\end{array}$ & $\begin{array}{l}-0.0008 \\
(0.60)\end{array}$ & $\begin{array}{l}0.0068 \\
(0.83)\end{array}$ \\
\hline Muette Afrique & $\begin{array}{l}-0.062 \\
(3.78)^{* * *}\end{array}$ & $\begin{array}{l}-0.070 \\
(3.50)^{* * *}\end{array}$ & $\begin{array}{l}-0.070 \\
(3.19)^{* * *}\end{array}$ & $\begin{array}{l}-0.065 \\
(2.89)^{* * *}\end{array}$ & $\begin{array}{l}-0.055 \\
(2.53)^{* *}\end{array}$ & $\begin{array}{l}-0.038 \\
(1.74)^{*}\end{array}$ & $\begin{array}{l}-0.015 \\
(0.66)\end{array}$ & $\begin{array}{l}0.009 \\
(0.39)\end{array}$ & $\begin{array}{l}0.057 \\
(2.10)^{* *}\end{array}$ & $\begin{array}{l}0.285 \\
(1.70)^{*}\end{array}$ \\
\hline Muette Amérique latine & $\begin{array}{l}-0.132 \\
(8.19)^{* * *}\end{array}$ & $\begin{array}{l}-0.135 \\
(7.27)^{* * *}\end{array}$ & $\begin{array}{l}-0.128 \\
(6.50)^{* * *}\end{array}$ & $\begin{array}{l}-0.117 \\
(5.69)^{* * *}\end{array}$ & $\begin{array}{l}-0.103 \\
(4.94)^{* * *}\end{array}$ & $\begin{array}{l}-0.088 \\
(4.28)^{* * *}\end{array}$ & $\begin{array}{l}-0.061 \\
(3.22)^{* * *}\end{array}$ & $\begin{array}{l}-0.021 \\
(1.23)\end{array}$ & $\begin{array}{l}0.070 \\
(2.98)^{* * *}\end{array}$ & $\begin{array}{l}0.711 \\
(5.00)^{* * *}\end{array}$ \\
\hline Constant & $\begin{array}{l}0.369 \\
(14.87)^{* * *}\end{array}$ & $\begin{array}{l}0.461 \\
(16.47) * * *\end{array}$ & $\begin{array}{l}0.554 \\
(18.26)^{* * *}\end{array}$ & $\begin{array}{l}0.646 \\
(19.96)^{* * *}\end{array}$ & $\begin{array}{l}0.732 \\
(21.79) * * *\end{array}$ & $\begin{array}{l}0.823 \\
(23.69) * * *\end{array}$ & $\begin{array}{l}0.940 \\
(26.41)^{* * *}\end{array}$ & $\begin{array}{l}1.117 \\
(30.91)^{* * *}\end{array}$ & $\begin{array}{l}1.411 \\
(30.00)^{* * *}\end{array}$ & $\begin{array}{l}3.012 \\
(12.45)^{* * *}\end{array}$ \\
\hline Observations & 148 & 151 & 151 & 151 & 151 & 151 & 151 & 151 & 151 & 151 \\
\hline Hansen p-value & 0.66 & 0.93 & 0.87 & 0.72 & 0.61 & 0.53 & 0.37 & 0.30 & 0.11 & 0.73 \\
\hline
\end{tabular}


Tableau A 3 : Influence des flux de financement en fonction du caractère démocratique, 1988-1998, 64 pays

\begin{tabular}{|c|c|c|c|c|c|c|c|c|c|c|}
\hline & $\begin{array}{c}1^{\mathrm{er}} \text { décile } \\
(1)\end{array}$ & $\begin{array}{c}2^{\text {ème }} \text { décile } \\
(2)\end{array}$ & $\begin{array}{c}3^{\text {ème }} \text { décile } \\
\text { (3) }\end{array}$ & $\begin{array}{c}4^{\text {ème }} \text { décile } \\
\text { (4) }\end{array}$ & $\begin{array}{c}5^{\text {ème }} \text { décile } \\
(5)\end{array}$ & $\begin{array}{c}6^{\text {ème }} \text { décile } \\
(6)\end{array}$ & $\begin{array}{c}7^{\text {ème }} \text { décile } \\
(7)\end{array}$ & $\begin{array}{c}8^{\text {ème }} \text { décile } \\
(8)\end{array}$ & $\begin{array}{c}9^{\text {ème }} \text { décile } \\
(9)\end{array}$ & $\begin{array}{c}10^{\text {ème }} \text { décile } \\
\text { (10) }\end{array}$ \\
\hline Ouverture (\% du PIB) & $\begin{array}{l}-0.083 \\
(1.57)\end{array}$ & $\begin{array}{l}-0.133 \\
(2.03)^{* *}\end{array}$ & $\begin{array}{l}-0.135 \\
(2.07)^{* *}\end{array}$ & $\begin{array}{l}-0.135 \\
(2.11)^{* *}\end{array}$ & $\begin{array}{l}-0.130 \\
(2.08)^{* *}\end{array}$ & $\begin{array}{l}-0.098 \\
(1.70)^{*}\end{array}$ & $\begin{array}{l}-0.068 \\
(1.29)\end{array}$ & $\begin{array}{l}0.024 \\
(0.50)\end{array}$ & $\begin{array}{l}0.121 \\
(2.03)^{* *}\end{array}$ & $\begin{array}{l}0.730 \\
(1.74)^{*}\end{array}$ \\
\hline Ouverture x Revenu p.c & $\begin{array}{l}0.020 \\
(1.97)^{* *}\end{array}$ & $\begin{array}{l}0.030 \\
(2.53)^{* *}\end{array}$ & $\begin{array}{l}0.029 \\
(2.47)^{* *}\end{array}$ & $\begin{array}{l}0.030 \\
(2.52) * *\end{array}$ & $\begin{array}{l}0.030 \\
(2.56)^{* *}\end{array}$ & $\begin{array}{l}0.026 \\
(2.38)^{* *}\end{array}$ & $\begin{array}{l}0.023 \\
(2.27)^{* *}\end{array}$ & $\begin{array}{l}0.007 \\
(0.75)\end{array}$ & $\begin{array}{l}-0.023 \\
(1.92)^{*}\end{array}$ & $\begin{array}{l}-0.196 \\
(2.54)^{* *}\end{array}$ \\
\hline IDE (\% du PIB) & $\begin{array}{l}0.011 \\
(0.01)\end{array}$ & $\begin{array}{l}0.816 \\
(0.56)\end{array}$ & $\begin{array}{l}0.982 \\
(0.67)\end{array}$ & $\begin{array}{l}1.035 \\
(0.71)\end{array}$ & $\begin{array}{l}1.264 \\
(0.90)\end{array}$ & $\begin{array}{l}1.393 \\
(1.06)\end{array}$ & $\begin{array}{l}1.885 \\
(1.51)\end{array}$ & $\begin{array}{l}1.287 \\
(1.02)\end{array}$ & $\begin{array}{l}0.295 \\
(0.17)\end{array}$ & $\begin{array}{l}-13.102 \\
(1.34)\end{array}$ \\
\hline IDE x “Démocratie” & $\begin{array}{l}-4.908 \\
(2.21)^{* *}\end{array}$ & $\begin{array}{l}-6.492 \\
(2.44)^{* *}\end{array}$ & $\begin{array}{l}-6.671 \\
(2.43)^{* *}\end{array}$ & $\begin{array}{l}-6.748 \\
(2.43)^{* *}\end{array}$ & $\begin{array}{l}-6.891 \\
(2.51)^{* *}\end{array}$ & $\begin{array}{l}-6.796 \\
(2.60)^{* * *}\end{array}$ & $\begin{array}{l}-6.924 \\
(2.88)^{* * *}\end{array}$ & $\begin{array}{l}-4.303 \\
(1.95)^{*}\end{array}$ & $\begin{array}{l}2.975 \\
(1.00)\end{array}$ & $\begin{array}{l}52.708 \\
(2.90)^{* * *}\end{array}$ \\
\hline Aide (\% du PIB) & $\begin{array}{l}-0.585 \\
(1.85)^{*}\end{array}$ & $\begin{array}{l}-0.514 \\
(1.37)\end{array}$ & $\begin{array}{l}-0.563 \\
(1.45)\end{array}$ & $\begin{array}{l}-0.641 \\
(1.61)\end{array}$ & $\begin{array}{l}-0.614 \\
(1.56)\end{array}$ & $\begin{array}{l}-0.534 \\
(1.44)\end{array}$ & $\begin{array}{l}-0.390 \\
(1.14)\end{array}$ & $\begin{array}{l}-0.084 \\
(0.30)\end{array}$ & $\begin{array}{l}0.551 \\
(1.72)^{*}\end{array}$ & $\begin{array}{l}3.887 \\
(1.52)\end{array}$ \\
\hline Aide x “Démocratie" & $\begin{array}{l}0.834 \\
(2.27)^{* *}\end{array}$ & $\begin{array}{l}0.863 \\
(2.15)^{* *}\end{array}$ & $\begin{array}{l}0.906 \\
(2.25)^{* *}\end{array}$ & $\begin{array}{l}0.968 \\
(2.40)^{* *}\end{array}$ & $\begin{array}{l}0.948 \\
(2.36)^{* *}\end{array}$ & $\begin{array}{l}0.838 \\
(2.17)^{* *}\end{array}$ & $\begin{array}{l}0.692 \\
(1.91)^{*}\end{array}$ & $\begin{array}{l}0.237 \\
(0.76)\end{array}$ & $\begin{array}{l}-0.625 \\
(2.07)^{* *}\end{array}$ & $\begin{array}{l}-6.040 \\
(2.20)^{* *}\end{array}$ \\
\hline Transferts (\% du PIB) & $\begin{array}{l}1.535 \\
(3.45)^{* * *}\end{array}$ & $\begin{array}{l}1.667 \\
(3.43)^{* * *}\end{array}$ & $\begin{array}{l}1.492 \\
(3.12)^{* * *}\end{array}$ & $\begin{array}{l}1.243 \\
(2.67)^{* * *}\end{array}$ & $\begin{array}{l}1.007 \\
(2.25)^{* *}\end{array}$ & $\begin{array}{l}0.791 \\
(2.13)^{* *}\end{array}$ & $\begin{array}{l}0.813 \\
(2.16)^{* *}\end{array}$ & $\begin{array}{l}0.340 \\
(0.60)\end{array}$ & $\begin{array}{l}-0.037 \\
(0.05)\end{array}$ & $\begin{array}{l}-8.890 \\
(3.99)^{* * *}\end{array}$ \\
\hline Transferts x "Démocratie" & $\begin{array}{l}-1.2691 \\
(1.40)\end{array}$ & $\begin{array}{l}-1.5079 \\
(1.41)\end{array}$ & $\begin{array}{l}-1.4373 \\
(1.29)\end{array}$ & $\begin{array}{l}-1.1526 \\
(1.06)\end{array}$ & $\begin{array}{l}-1.0510 \\
(0.98)\end{array}$ & $\begin{array}{l}-0.9596 \\
(1.03)\end{array}$ & $\begin{array}{l}-1.5541 \\
(1.68)^{*}\end{array}$ & $\begin{array}{l}-1.0550 \\
(1.11)\end{array}$ & $\begin{array}{l}-0.7397 \\
(0.59)\end{array}$ & $\begin{array}{l}12.4347 \\
(1.94)^{*}\end{array}$ \\
\hline Muette "Démocratie" & $\begin{array}{l}0.104 \\
(1.80)^{*}\end{array}$ & $\begin{array}{l}0.161 \\
(2.19)^{* *}\end{array}$ & $\begin{array}{l}0.162 \\
(2.08)^{* *}\end{array}$ & $\begin{array}{l}0.158 \\
(1.96)^{* *}\end{array}$ & $\begin{array}{l}0.156 \\
(1.93)^{*}\end{array}$ & $\begin{array}{l}0.145 \\
(1.87)^{*}\end{array}$ & $\begin{array}{l}0.146 \\
(1.99)^{* *}\end{array}$ & $\begin{array}{l}0.064 \\
(0.97)\end{array}$ & $\begin{array}{l}-0.103 \\
(1.25)\end{array}$ & $\begin{array}{l}-1.158 \\
(2.23)^{* *}\end{array}$ \\
\hline Revenu p.c. & $\begin{array}{l}-0.025 \\
(2.25)^{* *}\end{array}$ & $\begin{array}{l}-0.021 \\
(1.52)\end{array}$ & $\begin{array}{l}-0.023 \\
(1.52)\end{array}$ & $\begin{array}{l}-0.025 \\
(1.54)\end{array}$ & $\begin{array}{l}-0.023 \\
(1.40)\end{array}$ & $\begin{array}{l}-0.018 \\
(1.14)\end{array}$ & $\begin{array}{l}-0.008 \\
(0.54)\end{array}$ & $\begin{array}{l}0.007 \\
(0.53)\end{array}$ & $\begin{array}{l}0.044 \\
(3.16)^{* * *}\end{array}$ & $\begin{array}{l}0.121 \\
(1.11)\end{array}$ \\
\hline Revenu p.c. au carré & $\begin{array}{l}0.0007 \\
(0.69)\end{array}$ & $\begin{array}{l}0.0000 \\
(0.04)\end{array}$ & $\begin{array}{l}0.0003 \\
(0.26)\end{array}$ & $\begin{array}{l}0.0005 \\
(0.37)\end{array}$ & $\begin{array}{l}0.0004 \\
(0.32)\end{array}$ & $\begin{array}{l}0.0003 \\
(0.25)\end{array}$ & $\begin{array}{l}-0.0003 \\
(0.25)\end{array}$ & $\begin{array}{l}-0.0005 \\
(0.54)\end{array}$ & $\begin{array}{l}-0.0016 \\
(1.42)\end{array}$ & $\begin{array}{l}0.0002 \\
(0.03)\end{array}$ \\
\hline Muette Afrique & $\begin{array}{l}-0.015 \\
(0.48)\end{array}$ & $\begin{array}{l}-0.003 \\
(0.08)\end{array}$ & $\begin{array}{l}0.001 \\
(0.02)\end{array}$ & $\begin{array}{l}0.015 \\
(0.35)\end{array}$ & $\begin{array}{l}0.022 \\
(0.53)\end{array}$ & $\begin{array}{l}0.023 \\
(0.58)\end{array}$ & $\begin{array}{l}0.036 \\
(0.93)\end{array}$ & $\begin{array}{l}0.017 \\
(0.45)\end{array}$ & $\begin{array}{l}-0.023 \\
(0.61)\end{array}$ & $\begin{array}{l}-0.136 \\
(0.52)\end{array}$ \\
\hline Muette Amérique latine & $\begin{array}{l}-0.127 \\
(6.02)^{* * *}\end{array}$ & $\begin{array}{l}-0.137 \\
(5.51)^{* * *}\end{array}$ & $\begin{array}{l}-0.128 \\
(4.92)^{* * *}\end{array}$ & $\begin{array}{l}-0.115 \\
(4.33)^{* * *}\end{array}$ & $\begin{array}{l}-0.099 \\
(3.78)^{* * *}\end{array}$ & $\begin{array}{l}-0.083 \\
(3.31)^{* * *}\end{array}$ & $\begin{array}{l}-0.055 \\
(2.46)^{* *}\end{array}$ & $\begin{array}{l}-0.009 \\
(0.52)\end{array}$ & $\begin{array}{l}0.078 \\
(3.95)^{* * *}\end{array}$ & $\begin{array}{l}0.680 \\
(3.80)^{* * *}\end{array}$ \\
\hline Constant & $\begin{array}{l}0.326 \\
(7.29)^{* * *}\end{array}$ & $\begin{array}{l}0.422 \\
(7.86)^{* * *}\end{array}$ & $\begin{array}{l}0.522 \\
(9.22)^{* * *}\end{array}$ & $\begin{array}{l}0.617 \\
(10.50)^{* * *}\end{array}$ & $\begin{array}{l}0.707 \\
(12.08)^{* * *}\end{array}$ & $\begin{array}{l}0.805 \\
(14.61)^{* * *}\end{array}$ & $\begin{array}{l}0.913 \\
(18.61)^{* * *}\end{array}$ & $\begin{array}{l}1.099 \\
(28.26)^{* * *}\end{array}$ & $\begin{array}{l}1.418 \\
(27.60) * * *\end{array}$ & $\begin{array}{l}3.142 \\
(8.42) * * *\end{array}$ \\
\hline Observations & 148 & 151 & 151 & 151 & 151 & 151 & 151 & 151 & 151 & 151 \\
\hline Hansen p-value & 0.87 & 0.94 & 0.95 & 0.94 & 0.89 & 0.84 & 0.66 & 0.32 & 0.06 & 0.97 \\
\hline
\end{tabular}


Tableau A 4 : Existe-t-il une spécificité africaine dans la relation aide-inégalités ? 1988-1998, 64 pays

\begin{tabular}{|c|c|c|c|c|c|c|c|c|c|c|}
\hline & $\begin{array}{l}1^{\mathrm{er}} \text { décile } \\
(1)\end{array}$ & $\begin{array}{l}2^{\text {ème }} \text { décile } \\
\text { (2) }\end{array}$ & $\begin{array}{l}3^{\text {ème }} \text { décile } \\
\text { (3) }\end{array}$ & $\begin{array}{l}4^{\text {ème }} \text { décile } \\
\text { (4) }\end{array}$ & $\begin{array}{c}5^{\text {ème }} \text { décile } \\
\text { (5) }\end{array}$ & $\begin{array}{l}6^{\text {ème }} \text { décile } \\
\text { (6) }\end{array}$ & $\begin{array}{c}7^{\text {ème }} \text { décile } \\
(7)\end{array}$ & $\begin{array}{c}8^{\text {ème }} \text { décile } \\
\text { (8) }\end{array}$ & $\begin{array}{l}9^{\text {ème }} \text { décile } \\
\text { (9) }\end{array}$ & $\begin{array}{c}10^{\text {ème }} \text { décile } \\
\text { (10) }\end{array}$ \\
\hline Ouverture (\% du PIB) & $\begin{array}{l}-0.088 \\
(1.95)^{*}\end{array}$ & $\begin{array}{l}-0.101 \\
(1.75)^{*}\end{array}$ & $\begin{array}{l}-0.092 \\
(1.54)\end{array}$ & $\begin{array}{l}-0.093 \\
(1.55)\end{array}$ & $\begin{array}{l}-0.091 \\
(1.56)\end{array}$ & $\begin{array}{l}-0.066 \\
(1.13)\end{array}$ & $\begin{array}{l}-0.040 \\
(0.73)\end{array}$ & $\begin{array}{l}0.065 \\
(1.35)\end{array}$ & $\begin{array}{l}0.166 \\
(2.95)^{* * *}\end{array}$ & $\begin{array}{l}0.511 \\
(1.29)\end{array}$ \\
\hline Ouverture x Revenu p.c & $\begin{array}{l}0.012 \\
(1.87)^{*}\end{array}$ & $\begin{array}{l}0.014 \\
(1.77)^{*}\end{array}$ & $\begin{array}{l}0.013 \\
(1.60)\end{array}$ & $\begin{array}{l}0.014 \\
(1.73)^{*}\end{array}$ & $\begin{array}{l}0.014 \\
(1.77)^{*}\end{array}$ & $\begin{array}{l}0.011 \\
(1.38)\end{array}$ & $\begin{array}{l}0.007 \\
(0.92)\end{array}$ & $\begin{array}{l}-0.006 \\
(0.75)\end{array}$ & $\begin{array}{l}-0.023 \\
(2.47)^{* *}\end{array}$ & $\begin{array}{l}-0.076 \\
(1.44)\end{array}$ \\
\hline IDE (\% du PIB) & $\begin{array}{l}-1.125 \\
(1.95)^{*}\end{array}$ & $\begin{array}{l}-1.117 \\
(1.59)\end{array}$ & $\begin{array}{l}-1.361 \\
(1.81)^{*}\end{array}$ & $\begin{array}{l}-1.585 \\
(2.03)^{* *}\end{array}$ & $\begin{array}{l}-1.594 \\
(2.01)^{* *}\end{array}$ & $\begin{array}{l}-1.665 \\
(2.06)^{* *}\end{array}$ & $\begin{array}{l}-1.495 \\
(1.86)^{*}\end{array}$ & $\begin{array}{l}-1.433 \\
(1.75)^{*}\end{array}$ & $\begin{array}{l}0.228 \\
(0.23)\end{array}$ & $\begin{array}{l}11.100 \\
(1.92)^{*}\end{array}$ \\
\hline Aide (\% du PIB) & $\begin{array}{l}-0.086 \\
(0.30)\end{array}$ & $\begin{array}{l}-0.047 \\
(0.14)\end{array}$ & $\begin{array}{l}-0.112 \\
(0.28)\end{array}$ & $\begin{array}{l}-0.076 \\
(0.18)\end{array}$ & $\begin{array}{l}0.025 \\
(0.06)\end{array}$ & $\begin{array}{l}0.165 \\
(0.37)\end{array}$ & $\begin{array}{l}0.328 \\
(0.79)\end{array}$ & $\begin{array}{l}0.349 \\
(0.91)\end{array}$ & $\begin{array}{l}-0.200 \\
(0.53)\end{array}$ & $\begin{array}{l}-1.570 \\
(0.50)\end{array}$ \\
\hline Aide x “Afrique” & $\begin{array}{l}-0.210 \\
(0.68)\end{array}$ & $\begin{array}{l}-0.242 \\
(0.65)\end{array}$ & $\begin{array}{l}-0.207 \\
(0.48)\end{array}$ & $\begin{array}{l}-0.227 \\
(0.48)\end{array}$ & $\begin{array}{l}-0.261 \\
(0.54)\end{array}$ & $\begin{array}{l}-0.387 \\
(0.79)\end{array}$ & $\begin{array}{l}-0.469 \\
(1.00)\end{array}$ & $\begin{array}{l}-0.549 \\
(1.24)\end{array}$ & $\begin{array}{l}0.159 \\
(0.30)\end{array}$ & $\begin{array}{l}3.267 \\
(0.97)\end{array}$ \\
\hline Transferts (\% du PIB) & $\begin{array}{l}0.888 \\
(3.14)^{* * *}\end{array}$ & $\begin{array}{l}1.128 \\
(3.42)^{* * *}\end{array}$ & $\begin{array}{l}1.062 \\
(3.16)^{* * *}\end{array}$ & $\begin{array}{l}1.015 \\
(3.00)^{* * *}\end{array}$ & $\begin{array}{l}0.917 \\
(2.95)^{* * *}\end{array}$ & $\begin{array}{l}0.842 \\
(2.82)^{* * *}\end{array}$ & $\begin{array}{l}0.582 \\
(1.68)^{*}\end{array}$ & $\begin{array}{l}-0.012 \\
(0.03)\end{array}$ & $\begin{array}{l}-0.633 \\
(1.20)\end{array}$ & $\begin{array}{l}-7.460 \\
(3.27)^{* * *}\end{array}$ \\
\hline Revenu p.c. & $\begin{array}{l}-0.026 \\
(3.58)^{* * *}\end{array}$ & $\begin{array}{l}-0.025 \\
(3.02)^{* * *}\end{array}$ & $\begin{array}{l}-0.027 \\
(2.85)^{* * *}\end{array}$ & $\begin{array}{l}-0.024 \\
(2.37)^{* *}\end{array}$ & $\begin{array}{l}-0.019 \\
(1.74)^{*}\end{array}$ & $\begin{array}{l}-0.010 \\
(0.93)\end{array}$ & $\begin{array}{l}0.002 \\
(0.18)\end{array}$ & $\begin{array}{l}0.0110 \\
(0.93)\end{array}$ & $\begin{array}{l}0.032 \\
(2.20)^{* *}\end{array}$ & $\begin{array}{l}0.057 \\
(0.75)\end{array}$ \\
\hline Revenu p.c. au carré & $\begin{array}{l}0.001 \\
(2.20)^{* *}\end{array}$ & $\begin{array}{l}0.001 \\
(1.82)^{*}\end{array}$ & $\begin{array}{l}0.002 \\
(2.06)^{* *}\end{array}$ & $\begin{array}{l}0.001 \\
(1.77)^{*}\end{array}$ & $\begin{array}{l}0.001 \\
(1.32)\end{array}$ & $\begin{array}{l}0.001 \\
(0.91)\end{array}$ & $\begin{array}{l}0.000 \\
(0.19)\end{array}$ & $\begin{array}{l}0.000 \\
(0.03)\end{array}$ & $\begin{array}{l}-0.001 \\
(0.96)\end{array}$ & $\begin{array}{l}-0.004 \\
(0.73)\end{array}$ \\
\hline Muette Afrique & $\begin{array}{l}-0.046 \\
(2.12)^{* *}\end{array}$ & $\begin{array}{l}-0.054 \\
(2.13)^{* *}\end{array}$ & $\begin{array}{l}-0.053 \\
(1.90)^{*}\end{array}$ & $\begin{array}{l}-0.044 \\
(1.50)\end{array}$ & $\begin{array}{l}-0.033 \\
(1.13)\end{array}$ & $\begin{array}{l}-0.010 \\
(0.37)\end{array}$ & $\begin{array}{l}0.017 \\
(0.59)\end{array}$ & $\begin{array}{l}0.045 \\
(1.49)\end{array}$ & $\begin{array}{l}0.054 \\
(1.29)\end{array}$ & $\begin{array}{l}0.038 \\
(0.18)\end{array}$ \\
\hline Muette Amérique latine & $\begin{array}{l}-0.136 \\
(8.70)^{* * *}\end{array}$ & $\begin{array}{l}-0.142 \\
(7.67)^{* * *}\end{array}$ & $\begin{array}{l}-0.130 \\
(6.65)^{* * *}\end{array}$ & $\begin{array}{l}-0.117 \\
(5.72)^{* * *}\end{array}$ & $\begin{array}{l}-0.103 \\
(4.98)^{* * *}\end{array}$ & $\begin{array}{l}-0.089 \\
(4.40)^{* * *}\end{array}$ & $\begin{array}{l}-0.066 \\
(3.58)^{* * *}\end{array}$ & $\begin{array}{l}-0.022 \\
(1.32)\end{array}$ & $\begin{array}{l}0.083 \\
(4.15)^{* * *}\end{array}$ & $\begin{array}{l}0.728 \\
(5.47)^{* * *}\end{array}$ \\
\hline Constant & $\begin{array}{l}0.381 \\
(17.67) * * *\end{array}$ & $\begin{array}{l}0.502 \\
(21.96)^{* * *}\end{array}$ & $\begin{array}{l}0.598 \\
(22.16)^{* * *}\end{array}$ & $\begin{array}{l}0.682 \\
(22.31)^{* * *}\end{array}$ & $\begin{array}{l}0.760 \\
(23.37)^{* * * *}\end{array}$ & $\begin{array}{l}0.840 \\
(24.67) * * *\end{array}$ & $\begin{array}{l}0.941 \\
(26.82) * * *\end{array}$ & $\begin{array}{l}1.099 \\
(29.63) * * *\end{array}$ & $\begin{array}{l}1.398 \\
(31.08) * * *\end{array}$ & $\begin{array}{l}2.897 \\
(11.62) * * *\end{array}$ \\
\hline Observations & 148 & 151 & 151 & 151 & 151 & 151 & 151 & 151 & 151 & 151 \\
\hline Hansen p-value & 0.53 & 0.68 & 0.55 & 0.41 & 0.32 & 0.39 & 0.31 & 0.35 & 0.10 & 0.56 \\
\hline
\end{tabular}

Estimations par MMG (programme ivreg2.do développé par Baum et al. (2003)). Estimations robustes à l’hétéroscédasticité; * significatif à 10\%; ** à 5\%; *** à 1\%. 